\title{
Improved interior wall detection using designated dictionaries in compressive urban sensing problems
}

\author{
Eva Lagunas ${ }^{a}$, Moeness G. Amin ${ }^{\ddagger}$, Fauzia Ahmad $^{b}$ and Montse Nájar ${ }^{a}$ \\ ${ }^{a}$ Signal Theory and Communications Department, Universitat Politècnica de Catalunya, \\ Barcelona, 08034 Spain; \\ ${ }^{b}$ Radar Imaging Lab, Center for Advanced Communications, Villanova University, Villanova, \\ PA 19085, USA.
}

\begin{abstract}
In this paper, we address sparsity-based imaging of building interior structures for through-the-wall radar imaging and urban sensing applications. The proposed approach utilizes information about common building construction practices to form an appropriate sparse representation of the building layout. With a ground based SAR system, and considering that interior walls are either parallel or perpendicular to the exterior walls, the antenna at each position would receive reflections from the walls parallel to the radar's scan direction as well as from the corners between two meeting walls. We propose a two-step approach for wall detection and localization. In the first step, a dictionary of possible wall locations is used to recover the positions of both interior and exterior walls that are parallel to the scan direction. A follow-on step uses a dictionary of possible corner reflectors to locate wall-wall junctions along the detected wall segments, thereby determining the true wall extents and detecting walls perpendicular to the scan direction. The utility of the proposed approach is demonstrated using simulated data.
\end{abstract}

Keywords: Through-the-Wall Radar Imaging, Compressive Sensing, Building layout

\section{INTRODUCTION}

Through-the-wall Radar Imaging (TWRI) is an emerging technology that provides vision into building interiors from outside through exterior walls. ${ }^{1-3}$ This technology has numerous civilian and military applications, including search-and-rescue missions. ${ }^{4}$ In TWRI, radar returns from behind-the-wall scene are measured at several locations along a real or synthetic aperture, which are then processed to obtain high resolution scene images. High resolution imaging requires use of large bandwidth signals and long antenna arrays, which, in turn, implies acquisition and processing of large amounts of data. Compressive Sensing (CS) techniques have been applied recently to alleviate the data acquisition and processing bottlenecks. ${ }^{5-10}$ Besides improved data acquisition efficiency, use of reduced data volume in TWRI is important logistically, as some of the data observations in time, space, and frequency can be difficult or impossible to attain. Such difficulty may be attributed to interference within a frequency band or at specific frequencies, and also due to the inability of the electromagnetic (EM) waves to reach the behind-the-wall scene from certain aspect angles.

Recently, we addressed the problem of imaging building interior structures using a reduced number of measurements. ${ }^{11}$ Assuming a stepped-frequency, monostatic synthetic aperture radar (SAR) system, we devised a dictionary of possible wall locations for sparse representation, which is consistent with the fact that interior walls are typically parallel or perpendicular to the front wall. The dictionary accounted for the dominant normal angle reflections from exterior and interior walls for the monostatic imaging system. CS was applied to a reduced set of observations to recover the true positions of the walls. While the proposed method was successful in detecting interior walls that were parallel to the front wall, it could not determine the exact extents of the detected walls. Moreover, the proposed scheme could not locate walls that were perpendicular to the front wall, without access from another side of the building.

In this paper, we extend the method of Ref. 11 to overcome its aforementioned shortcomings by exploiting

$\ddagger$ moeness.amin@villanova.edu, http://www.villanova.edu/engineering/research/centers/cac/ 
the EM scatterings from corners created by the junction of parallel and perpendicular walls. As a follow-on step to the parallel wall detection scheme, we propose to use a sparsifying dictionary of possible corner reflectors, representing the reflection caused by the wall-wall junctions. CS technqiues are applied to the reduced set of observations to recover the positions of the corners along the previously detected parallel walls. Determining corner reflectors along the wall segments indicates the extents of the detected parallel walls and locates perpendicular walls. The proposed enhancement provides reliable determination of building interior layout while achieving substantial reduction in data volume.

Similar approaches were recently proposed in the literature. ${ }^{12-14}$ In Ref. 12, the Hough Transform (HT) domain for continuous infinite-length line detection was strictly discretized for tunnel detection in Ground Penetrating Radar (GPR). The TWRI problem, considered in Ref. 13, provided an improvement to the conventional HT reconstruction assuming knowledge of the orientation of walls and by applying sharp windowing to the resulting HT. Ref. 13, however, did not deal with a reduced data volume. In Ref. 14, a set of dictionaries corresponding to various dominant scattering phenomena were used for simultaneously reconstructing a set of sparse images of the scene, each indicating the location of respective scatterers. However, the employed dictionaries were based on linear and quadratic phase changes induced by the different scatterers.

The remainder of the paper is organized as follows. Section II reviews the wall detection approach presented in Ref. 11. Section III presents the sparsifying dictionary based on possible corner reflector locations for the follow-on step of revealing wall junctions. Supporting results based on simulated data are provided in Section IV. Conclusions are drawn in Section V.

\section{CS BASED INTERIOR WALL DETECTION}

\subsection{Linear model for interior wall detection}

Consider a SAR system in which a single antenna at one location transmits and receives the radar signal, then moves to the next location, and repeats the same operation parallel to a homogeneous wall, as shown in Fig. 1.

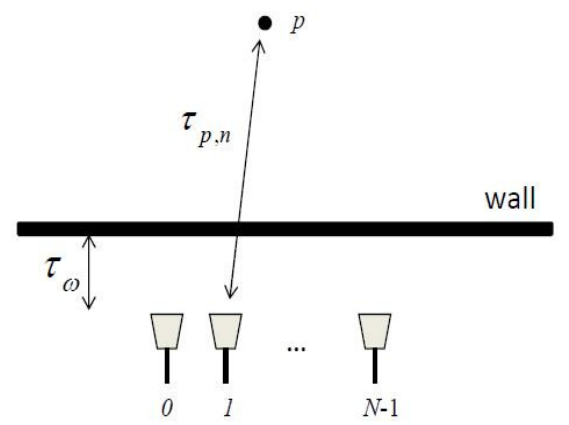

Figure 1. Data collection using a synthetic array in TWRI.

Assume $N$ antenna locations, which can be uniformly or randomly spaced. Since it is common practice to build interior walls either parallel or perpendicular to the front exterior wall, all interior walls present in the scene are assumed to be parallel or perpendicular to the array. Let the antenna at the $n$th location illuminate the scene with a stepped-frequency signal of $M$ frequencies, $\left\{\omega_{m}\right\}_{m=0}^{M-1}$, which are equispaced over the desired bandwidth $\omega_{M-1}-\omega_{0}$. Note that, due to the specular nature of the wall reflections, contributions from interior building walls only parallel to the antenna array will mostly be received at the array. For a scene consisting of $P$ point targets and $I_{w}$ interior walls parallel to the array axis, the signal received by the $n$th transceiver at the $m$ th frequency can be expressed as,

$$
y(m, n)=A_{w} e^{-j \omega_{m} \tau_{w}}+\sum_{i=0}^{I_{w}-1} A_{w_{i}} e^{-j \omega_{m} \tau_{w_{i}}}+\sum_{p=0}^{P-1} A_{p} e^{-j \omega_{m} \tau_{p, n}},
$$

In eqn. (1), $A_{w}, A_{w_{i}}$ and $A_{p}$ contain the amplitude of each scatterer. In general, each amplitude has three components, namely, the attenuation caused by the distance between the scatterer and the receiver, the attenuation 
caused by the propagation through the front wall, and the complex reflectivity of each scatterer. In the above equation, $\tau_{w}, \tau_{w_{i}}$ and $\tau_{p, n}$, respectively, represent the two-way traveling time of the signal from the $n$-th antenna to the wall, from the $n$th antenna to the $i$ th interior wall, and between the $n$th antenna and the $p$ th target. As the wall is a specular reflector and the antennas are located parallel to the front wall, the delays $\tau_{w}$ and $\tau_{w_{i}}$ are independent of the variable $n$, as evident in the subscripts. Given the exact knowledge of the wall permitivity and thickness, the effect of the front wall can be taken into account in $\tau_{w_{i}}$ and $\tau_{p, n} .{ }^{15-17}$

Let $\mathbf{y}_{n}=\left[\begin{array}{llll}y(0, n) & y(1, n) & \ldots & y(M-1, n)\end{array}\right]^{T}$ represent the received signal vector corresponding to the $M$ frequencies at the $n$th antenna location. Stacking the measurement vectors corresponding to all $N$ antennas forms the observation vector $\mathbf{y}$,

$$
\mathbf{y}=\left[\begin{array}{llll}
\mathbf{y}_{0}^{T} & \mathbf{y}_{1}^{T} & \cdots & \mathbf{y}_{N-1}^{T}
\end{array}\right]^{T}
$$

Assuming the region of interest is partitioned into a finite number of pixels, $N_{x} \times N_{z}$, in crossrange and downrange, the scene can be represented by the complex reflectivity function $r(k, l), k=0, \ldots, N_{x}-1, l=0, \ldots, N_{z}-1$. Let $\mathbf{r}$ be the concatenated $N_{x} N_{z} \times 1$ scene reflectivity vector corresponding to the spatial sampling grid. For the wall detection problem, the scene vector, $\mathbf{r}$, and the observation vector, $\mathbf{y}$, are related by

$$
\mathbf{y}=\hat{\mathbf{\Psi}} \mathbf{r}
$$

where the sensing matrix $\hat{\Psi}$ underlines the specular reflections produced by walls and is given by ${ }^{18}$

$$
\hat{\mathbf{\Psi}}=\left[\begin{array}{llll}
\hat{\mathbf{\Psi}}_{0}^{T} & \hat{\mathbf{\Psi}}_{1}^{T} & \cdots & \hat{\mathbf{\Psi}}_{N-1}^{T}
\end{array}\right]^{T}
$$

with $\hat{\mathbf{\Psi}}_{n}$ defined as,

$$
\left[\hat{\mathbf{\Psi}}_{n}\right]_{m}=\left[\Im_{[(00), n]} e^{-j \omega_{m} \tau_{(0,0)}} \quad \cdots \quad \Im_{\left[\left(N_{x}-1, N_{z}-1\right), n\right]} e^{-j \omega_{m} \tau_{\left(N_{x}-1, N_{z}-1\right)}}\right]
$$

In eqn. $(5), \tau_{(k, l)}=\frac{2 \rho_{l}}{c}$ is the two-way signal propagation time associated with the downrange $\rho_{l}$ of the $(k, l)$-th pixel, and the function $\Im_{[(k, l), n]}$ works as an indicator function in the following way,

$$
\Im_{[(k, l), n]}= \begin{cases}1 & \text { if the }(k, l) \text {-th pixel is in front of the } n \text {-th antenna } \\ 0 & \text { otherwise }\end{cases}
$$

The indicator function accounts for the fact that, due to wall specular reflections and since the array is assumed parallel to the front wall and, thus, parallel to interior walls, the rays collected at the $n$th antenna will be produced by portions of the walls that are only in front of that antenna. ${ }^{18}$

\subsection{Sparsifying basis for interior wall detection}

With the synthetic array aperture parallel to the front wall, interior walls parallel to the array will dominate the image. Focusing primarily on the detection of interior walls parallel to the front wall, we observe that the number of parallel walls is typically much smaller compared to the downrange extent of the building. As such, the decomposition of the image into horizontal walls can be considered as sparse. ${ }^{11}$ Note that although other indoor targets, such as furniture, humans and wall-wall corners, may be present in the scene, their projections onto the horizontal lines are expected to be negligible compared to those of the walls.

In order to obtain a linear matrix-vector relation between the scene and the horizontal projections, we define a sparsifying matrix $\mathbf{R}$ composed of possible wall locations. Specifically, each column of the dictionary $\mathbf{R}$ represents an image containing a single wall of length $l_{x}$ pixels, located at a specific crossrange and at a specific downrange in the image. Consider the crossrange to be divided into $N_{c}$ non-overlapping blocks of $l_{x}$ pixels each, and the downrange division defined by the pixel grid. The number of blocks $N_{c}$ is determined by the value of $l_{x}$, which is the minimum expected wall length in the scene. Therefore, the dimension of $\mathbf{R}$ is $N_{x} N_{z} \times N_{c} N_{z}$, where the product $N_{c} N_{z}$ denotes the number of possible wall locations. The projection associated with each wall location is given by,

$$
g^{(b)}(l)=\frac{1}{l_{x}} \sum_{k \in B[b]} r(k, l)
$$


where $B[b]$ indicates the $b$-th crossrange block and $b=1, \ldots, N_{c}$. Defining

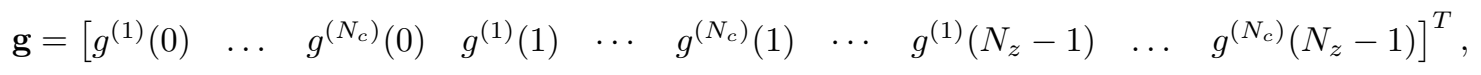

the linear system of equations relating the observed data $\mathbf{y}$ and the sparse vector $\mathbf{g}$ is given by,

$$
\mathbf{y}=\hat{\mathbf{\Psi}} \mathbf{R g}
$$

In practice and by the virtue of collecting signal reflections corresponding to the zero aspect angle, any interior wall outside the synthetic array extent will not be visible to the system.

\subsection{Sparse Scene Reconstruction}

Towards the objective of fast data acquisition, consider $\breve{\mathbf{y}}$, which is a vector of length $Q_{1} Q_{2}(<<M N)$, consisting of elements chosen from $\mathbf{y}$ as follows,

$$
\breve{\mathbf{y}}=\boldsymbol{\Phi} \mathbf{y}=\boldsymbol{\Phi} \hat{\mathbf{\Psi}} \mathbf{R g}
$$

where $\boldsymbol{\Phi}$ is a $Q_{1} Q_{2} \times M N$ matrix of the form,

$$
\boldsymbol{\Phi}=\operatorname{kron}\left(\boldsymbol{\vartheta}, \mathbf{I}_{Q_{1}}\right) \cdot \operatorname{diag}\left\{\boldsymbol{\varphi}^{(0)}, \ldots, \boldsymbol{\varphi}^{(N-1)}\right\} .
$$

In eqn. (11), 'kron' denotes the Kronecker product, $\vartheta$ is a $Q_{2} \times N$ measurement matrix constructed by uniformly or randomly selecting $Q_{2}$ rows of an $N \times N$ identity matrix, and $\varphi^{(n)}, n=0,1, \ldots, N-1$, is a $Q_{1} \times M$ measurement matrix constructed by uniformly or randomly selecting $Q_{1}$ rows of an $M \times M$ identity matrix. We note that $\boldsymbol{\vartheta}$ determines the reduced antenna locations, whereas $\varphi^{(n)}$ determines the reduced set of frequencies corresponding to the $n$th antenna location.

The reconstructed CS image is obtained by first recovering the projection vector $\mathbf{g}$ using Orthogonal Matching Pursuit (OMP) to solve the optimization problem,

$$
\min _{\mathbf{g}}\|\mathbf{g}\|_{l_{1}} \quad \text { subject to } \quad \breve{\mathbf{y}} \approx \mathbf{\Phi} \hat{\mathbf{\Psi}} \mathbf{R g}
$$

and then forming the product $\mathbf{R g}$.

It is noted that we are implicitly assuming that the extents of the walls in the scene are integer multiples of the block of $l_{x}$ pixels. In case this condition is not satisfied, the maximum error in determining the wall extent will be at the most equal to the chosen block size. In order to reduce this error, the chosen block size should be small. However, a smaller block size will cause the contributions from point-like targets to increase under the proposed sparsifying basis. In short, there exists a tradeoff between wall extent estimation error and the ability of the sparsifying basis to favor targets extended in crossrange. The proposed follow-on step of detecting reflections from wall-wall junctions can help alleviate this issue.

\section{PROPOSED EXTENSION BASED ON CORNER DETECTION}

Once the wall locations have been determined, more information about the walls and their extent can be inferred by detecting the corners (dihedral) formed by the intersection of a parallel wall and a perpendicular wall. Thus, the following corner detection approach should be considered as a follow-on step after the wall detection approach described in Section 2.

We first modify eqn. (1) to take into account the wall-wall junctions present in the scene. Considering the presence of $C$ corner reflectors, the signal received by the $n$th transceiver at the $m$ th frequency can be expressed as,

$$
y(m, n)=A_{w} e^{-j \omega_{m} \tau_{w}}+\sum_{i=0}^{I_{w}-1} A_{w_{i}} e^{-j \omega_{m} \tau_{w_{i}}}+\sum_{p=0}^{P-1} A_{p} e^{-j \omega_{m} \tau_{p, n}}+\sum_{q=0}^{C-1} \Upsilon_{[q, n]} A_{q} S_{m, q, n} e^{-j \omega_{m} \tau_{q, n}}
$$

with

$$
S_{m, q, n}=\operatorname{sinc}\left(\omega_{m} \frac{L_{q}}{c} \sin \left(\theta_{q, n}-\bar{\theta}_{q}\right)\right)
$$


The function $\Upsilon_{[q, n]}$ works as an indicator function in the following way,

$$
\Upsilon_{[q, n]}= \begin{cases}1 & \text { if } n \text {-th antenna illuminates the concave side of the } q \text { th corner } \\ 0 & \text { otherwise }\end{cases}
$$

In eqn. (13), $A_{q}$ contains the amplitude of each corner reflector, which includes the attenuation caused by the distance between the corner and the receiver, the attenuation caused by the propagation through the front wall, and the complex reflectivity of the corner reflector. The variables $L_{q}$ and $\bar{\theta}_{q}$, respectively, define the length and the orientation angle of the $q$ th corner reflector, whereas $\theta_{q, n}$ is the aspect angle associated with the $q$ th corner reflector and the $n$th antenna. In the above equation, $\tau_{q, n}$ represents the two-way traveling time of the signal between the $n$th antenna and the $q$ th corner.

Assuming that $N_{w}$ parallel walls have been previously detected, the sparsifying dictionary based on possible dihedral locations, named $\boldsymbol{\Lambda}$, can be introduced with $2 N_{x} N_{w}$ columns, each corresponding to a corner response, located at a different position with either $45^{\circ}$ or $135^{\circ}$ orientation angle. The corner response is modeled following eqn. (14). The linear relationship between the possible dihedral locations, represented by the column vector $\boldsymbol{\nu}$, and the reduced data measurements, $\breve{\mathbf{y}}$, is given by,

$$
\breve{\mathrm{y}}=\Phi \Lambda \nu
$$

Therefore, the initial step in the proposed algorithm is to detect wall positions from the compressed data measurements using the sparse model defined in eqn. (10). Using the downranges of the detected walls, a dictionary of dihedral positions (eqn. (16)) is then used to determine the extent of the walls.

\section{SIMULATION RESULTS}

In this section, we evaluate the performance of the proposed scheme using synthesized data. A stepped-frequency signal consisting of 335 frequencies covering the 1 to $2 \mathrm{GHz}$ frequency band was used for interrogating the scene. A monostatic synthetic aperture array, consisting of 71-element locations with an inter-element spacing of $2.2 \mathrm{~cm}$, was employed. The array was located parallel to a $1.6 \mathrm{~m}$-wide front wall, and centered at $0 \mathrm{~m}$ in crossrange at a standoff distance of $2.42 \mathrm{~m}$. The scene behind the front wall contained two interior walls, a back wall, and a single point target. The first interior wall extended from $-0.78 \mathrm{~m}$ to $-0.56 \mathrm{~m}$ in crossrange at a downrange of $3.37 \mathrm{~m}$, while the second wall was located at $5.12 \mathrm{~m}$ downrange, extending from $0.02 \mathrm{~m}$ to $0.78 \mathrm{~m}$ in crossrange. The $1.6 \mathrm{~m}$ wide back wall was located at $6.24 \mathrm{~m}$ and was aligned with the front wall in crossrange. To simulate different wall materials, we considered different reflectivities for interior $(\sigma=0.85)$ and exterior walls $(\sigma=1)$. The two interior walls and the back wall have corner reflectors on their extremities to emulate the junctions between parallel and perpendicular walls. The point target was located at $(0.02,4.24) \mathrm{m}$. Fig. 2 depicts the geometry of the simulated scene. The attenuation caused by the front wall was set to $10 \mathrm{~dB}$.

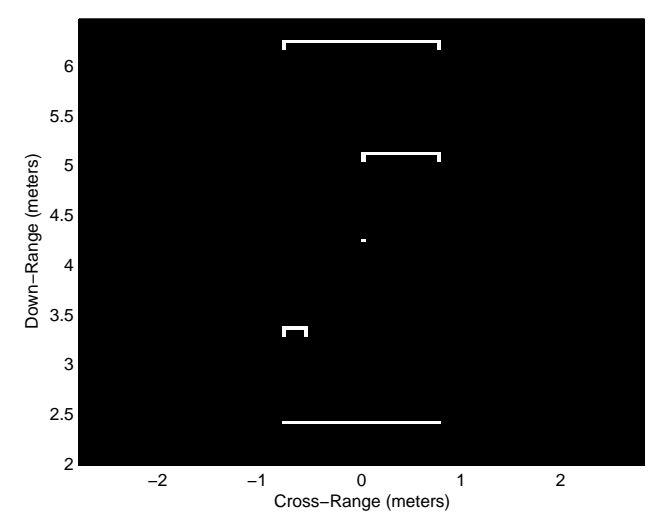

Figure 2. Geometry of the simulated scene 
The region to be imaged is chosen to be $5.65 \mathrm{~m}$ (crossrange) $\times 4.45 \mathrm{~m}$ (downrange), centered at $(0,4.23) \mathrm{m}$, and is divided into $128 \times 128$ pixels. The sparsifying matrix $\mathbf{R}$ contains predefined walls of size 1 pixel in downrange and 12 pixels in crossrange, which corresponds to a minimum wall length of $0.53 \mathrm{~m}$. We enforced the first interior wall to be half of the predefined wall block length in the dictionary $\mathbf{R}$, whereas the second interior wall occupies one and a half of the predefined wall blocks. For sparsity-based imaging, we consider only $6.4 \%$ of the full data volume (25\% uniformly selected frequencies and $25 \%$ uniformly chosen sensor locations). Fig. 3(a) and Fig. 3(b) show the recovered CS images from the wall detection step and the corner detection step, respectively. In these figures, we plot the image intensity with the maximum intensity value in each image normalized to 0dB. Fig. 3(a) shows how the wall that is occupying one and a half block is now recovered as a two block wall, and the half block wall is recovered as a complete block wall. Therefore, although the wall detection approach presented in ${ }^{11}$ has adequately detected the downrange position of the walls, it fails to correctly determine the corresponding wall extents. These initial wall estimates in Fig. 3(a) can be refined with the proposed corner detection approach, which accurately estimates the extent of each wal, as shown in Fig. 3(b).

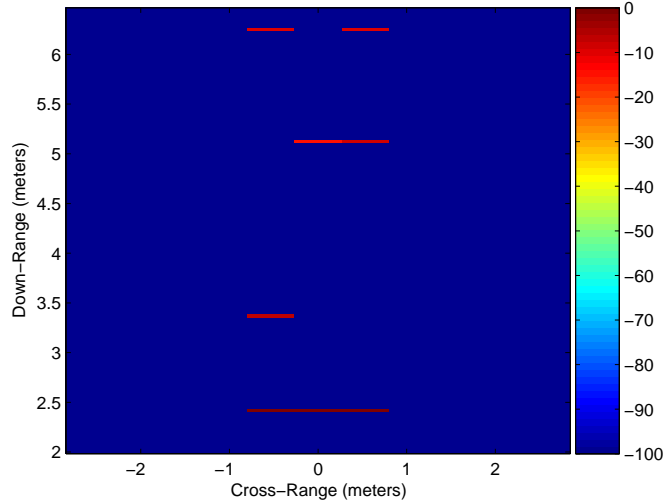

(a)

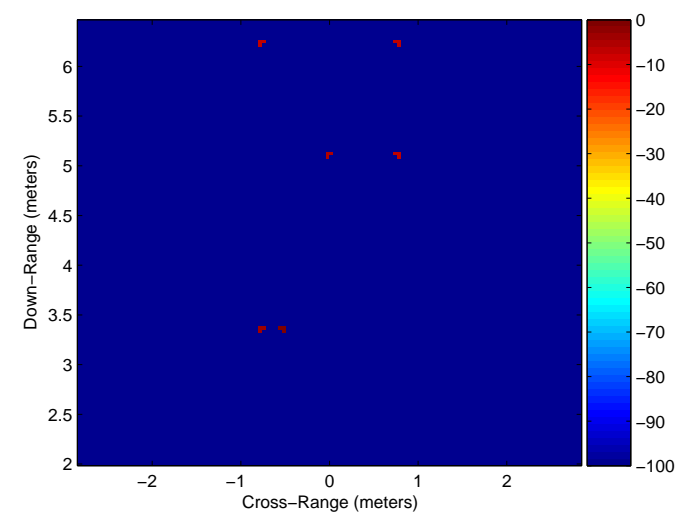

(b)

Figure 3. Reconstructed image from the recovered sparse vector using OMP and 6.4\% data: (a) Proposed approach for wall detection, (b) Proposed approach for corner detection.

\section{CONCLUSIONS}

A sparsity-based approach for imaging of building interior structures was presented. The proposed two-step approach makes use of prior information about building construction practices to form an appropriate sparse representation of the building interior layout. In the first step, a sparsifying dictionary based on the expected wall alignment relative to the radar's scan direction was designed and used to detect interior walls parallel to the front wall. In the second step, a sparsifying dictionary based on the possible corner reflector locations was employed to detect the wall-wall junctions along the detected parallel walls, in order to determine the wall extents and detect walls perpendicular to the front wall. The proposed method provides reliable determination of building layouts, while achieving substantial reduction in data volume. Results based on computer simulations were presented, which depicted the improved performance of the proposed method when compared to the wall-only detection approach of Ref. ${ }^{11}$

\section{ACKNOWLEDGMENTS}

The work by M. G. Amin and F. Ahmad was supported in part by ONR under grant N00014-11-1-0576 and in part by ARO and ARL under contract W911NF-11-1-0536. The work by E. Lagunas was performed during her tenure as a Visiting Research Associate at the Center for Advanced Communications, Villanova University, USA. E. Lagunas and M. Nájar are partially supported by the Spanish Ministry of Science and Innovation (Ministerio de Ciencia e Innovación) under project TEC2011-29006-C03-02 (GRE3N-LINK-MAC). 


\section{REFERENCES}

[1] Amin (Ed.), M. G., "Special issue on Advances in Indoor Radar Imaging," J. Franklin Inst. 345(6), 556-722 (Sep, 2008).

[2] Amin (Ed.), M. G., "Through-the-Wall Radar Imaging," CRC Press, Boca Raton, FL (2010).

[3] Amin, M. G. and Sarabandi (Eds.), K., "Special issue on Remote Sensing of Building Interior," IEEE Trans. Geosci. Remote Sens. 47(5), 1270-1420 (May, 2009).

[4] Burchett, H., "Advances in Through Wall Radar for Search, Rescue and Security Applications," Inst. of Eng. and Tech. Conf. Crime and Security, London, UK, 511-525 (Jun, 2006).

[5] Yoon, Y. and Amin, M. G., "Compressed Sensing Technique for High-Resolution Radar Imaging," Proc. SPIE 6968, 69681A-1-69681A-10 (2008).

[6] Huang, Q., Qu, L., Wu, B., and Fang, G., "UWB Through-Wall Imaging based on Compressive Sensing," IEEE Trans. Geosci. Remote Sens. 48(3), 1408-1415 (2010).

[7] Amin, M., Ahmad, F., and Zhang, W., "Target RCS exploitations in compressive sensing for through wall imaging," 5th Int. Waveform Diversity and Design Conf., Niagara Falls, Canada (Aug., 2010).

[8] Leigsnering, M., Debes, C., and Zoubir, A., "Compressive Sensing in Through-the-Wall Radar Imaging," IEEE Int. Conf. Acoustics, Speech and Signal Processing (ICASSP), Prague, Czech Republic, 4008-4011 (2011).

[9] Ahmad (Ed.), F., "Compressive Sensing," Proc. of SPIE, Bellingham, WA, 8365 (2012).

[10] Ahmad, F. and Amin, M., "Through-the-wall human motion indication using sparsity-driven change detection," IEEE Trans. Geosci. Remote Sens. 51(2), 881-890 (2013).

[11] Lagunas, E., Amin, M., Ahmad, F., and Najar, M., "Sparsity-Based Radar Imaging of Building Structures," European Signal Processing Conference (EUSIPCO), Bucharest, Romania (Aug, 2012).

[12] Gurbuz, A. C., McClellan, J., and Scott, W., "Compressive Sensing of underground structures using GPR," Digital Signal Processing 22(1), 66-73 (Jan, 2012).

[13] Aftanas, M. and Drutarovsky, M., "Imaging of the Building Contours with Through the Wall UWB Radar System," Radioengineering Journal 18(3), 258-264 (2009).

[14] Tan, R., de Wit, J., and van Rossum, W., "Radar mapping of building structures applying sparse reconstruction," 1st Int. Workshop on Compressed Sensing applied to Radar, Bonn, Germany (May, 2012).

[15] Ahmad, F., Amin, M. G., and Kassam, S. A., "A Beamforming Approach to Stepped-Frequency Synthetic Aperture Through-the-Wall Radar Imaging," IEEE Int. Workshop on Computational Advances in MultiSensor Adaptive Processing 345, 24-27 (2005).

[16] Ahmad, F. and Amin, M. G., "A noncoherent radar system approach for through-the-wall imaging," Proc. SPIE Symp. Defense and Security, Sensors, and Command, Control, Communications, and Intelligence (C3I) Technologies IV Conference 5778, 196-207 (2005).

[17] Amin, M. G. and Ahmad, F., "Wideband Synthetic Aperture Beamforming for Through-the-Wall Imaging," IEEE Signal Process. Mag. 25(4), 110-113 (Jul, 2008).

[18] Ahmad, F. and Amin, M. G., "Partially Sparse Reconstruction of Behind-the-Wall Scenes," Proc. SPIE Symp. Defense, Security, and Sensing, Compressive Sensing Conference, Baltimore, MD 8365 (Apr, 2012). 